\title{
Composite Recycling Techniques: A Literature Review
}

\section{Peng Hao Wang* and Natalie Zimmermann \\ School of Aviation and Transportation Technology, Purdue University, USA}

Submitted: February 07, 2020; Published: February 26, 2020

*Corresponding author: Peng Hao Wang, Assistant Professor, School of Aviation and Transportation Technology, Purdue University, West Lafayette, Indiana, USA

\begin{abstract}
Composite materials continue to grow in popularity in a variety of different industries around the world. One of these industries is the aerospace industry, where the application of composite materials has evolved from its limited utilization in secondary structures to today's complete primary and critical structures found in modern airliners. However, the increased utilization of composite materials has caused a surge in composite waste. As the global industry continues to develop additional applications for composite materials and pushing the boundaries thereof, the demand for virgin fibers and recycling solutions for used fibers will continue to rise. Thus, the proper management and recycling of composite materials may be a crucial aspect for the continued advancement and sustainability of composite materials in the aerospace industry. In this review, the authors examined various composite material recycling techniques currently being developed. The methodologies examined include thermal, chemical, and mechanical recycling techniques. This study aims to explore and provide an overview of the current state of the composite recycling industry as a reference for future development of existing and alternative recycling methodologies. .
\end{abstract}

Keywords: Composite materials; Fiberglass; Carbon fiber; Recycling; Aerospace

\section{Introduction}

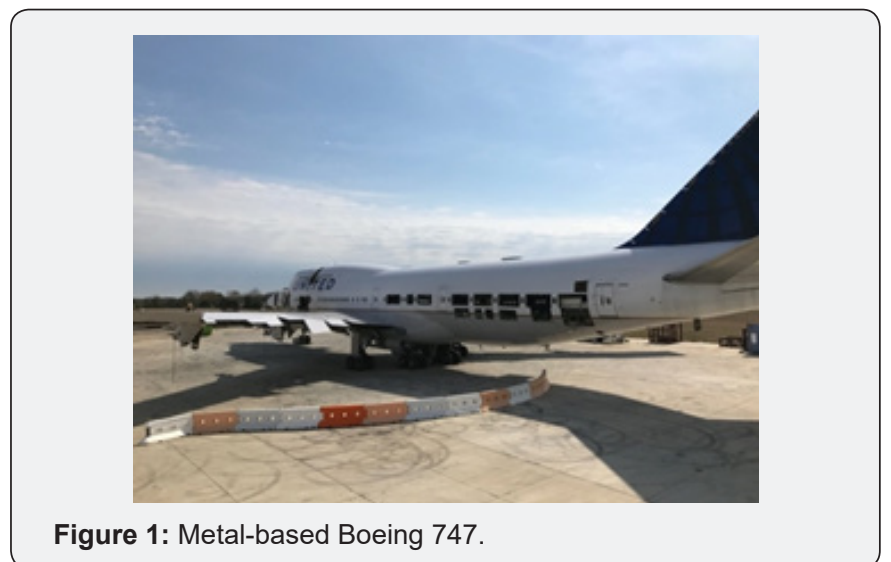

Composite materials have gained increased popularity for the manufacture of components and structures in various fields, such as in the aviation industry. However, with the increased use of composite materials, an increased amount of composite material waste is generated $[1,2]$ and new end-of-life (EoL) and disposability issues emerge [3]. For example, at the end of the useful life of an aircraft-as in the case of the Boeing 747 in Figure 1, usable and high-valued parts are sold or reused, while the airframe and the remaining components are disposed in landfills or incinerated $[1,3,4]$. However, this approach is not sustainable or highly viable for composite-based aircraft structures and components-such as the composite-based Boeing 787 in Figure 2, and presents a wide array of environmental concerns. First, the available landfill space is decreasing, while at the same time its cost is increasing [1] due to increased disposal and end-of-life regulations. Additionally, certain composite materials are classified as hazardous waste due to their high pollution characteristics, and as such cannot be disposed in landfills or incinerated at the end of their useful life without further treatment [3]. Furthermore, by disposing or incinerating composite materials, finite resources are being consumed [5] and valuable materials are destroyed [6].

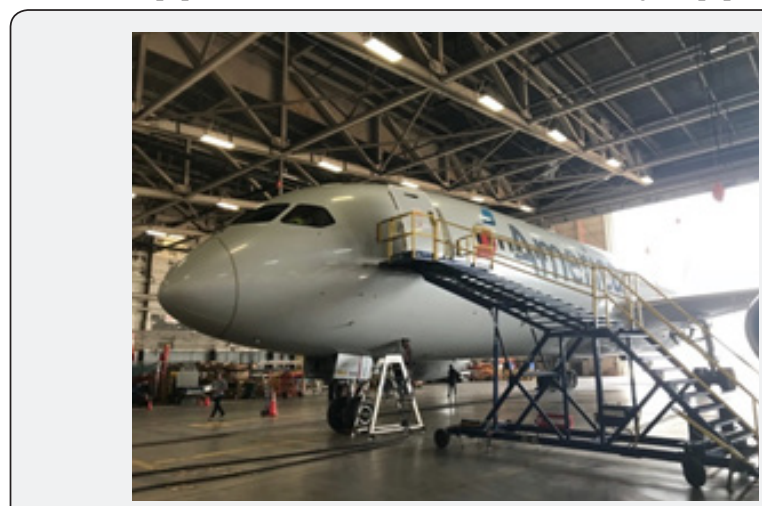

Figure 2: Composite-based Boeing 787. 
The recycling of composite materials at the end of their useful life is a possible alternative approach to landfilling and incineration, as it presents a wide array of environmental and economic advantages. The current demand of fiberglass and carbon fibers may lead to a shortage of virgin fibers [6]. By recycling and reusing composite materials, the expected fiber shortage can be potentially alleviated while at the same time reducing the environmental impact of current end-of-life methods. In addition, the manufacturing process of virgin composite materials is rather pricey, energy intensive, and results in a high number of emissions [7]. Using recycled fibers instead of virgin fibers to manufacture new components allows to reduce both the cost of, and energy consumed during the manufacturing process [3]. This study aims to explore and provide an overview of the current status of the composite recycling methodologies. Specifically, the literature of the thermal, chemical, and mechanical recycling processes is reviewed with the purpose of highlighting the current standing, advances, and limitations of the recycling methodologies researched.

\section{Discussion}

\section{Chemical recycling}

In the chemical recycling method, chemicals are used to degrade the organic polymers in composite materials, thus exposing the fillers and reinforcing fibers. The recovered fibers, fillers, and degraded organic materials have the potential to be reused in numerous applications. Depending on the solvent used for the chemical degradation, the chemical process can be divided into two categories: solvolysis and hydrolysis $[6,8,9]$.

\section{Solvolysis}

In the solvolysis process, organic solvents are used for the chemical degradation of the polymers $[6,8]$. The solvolysis process can be applied to both carbon fibers and glass fibers. However, the research on the application of the solvolysis process on carbon fibers is slightly more advanced than the research focused on recovering glass fibers [10]. Jiang et al. [11] studied the tensile, surface, and interfacial bonding characteristics of carbon fibers recovered through the use solvolysis via supercritical n-propanol. Samples of three different PAN based carbon fiber/epoxy resin composites were cut into $10 \mathrm{~mm} \times 200 \mathrm{~mm}$ pieces and placed in a semi-continuous flow reactor. n-propanol was pumped through the flow reactor to decompose the resin. The recovered fibers were cleaned in an ultrasound bath, rinsed with acetone, and dried at $80^{\circ} \mathrm{C}$ for a week. Consequently, the fibers were analyzed via scanning electron microscope (SEM), tensile tests, X-ray photon spectroscopy (XPS), and micro-droplet tests. Through the tests it was observed that the mechanical properties of the fibers remained unchanged, while the interfacial bonding strength with epoxy decreased due to the reduced surface oxygen [11]. Liu et al. [1] used a nitric acid bath to recover fibers from carbon/ epoxy composites. Both, the decomposed resin and the recovered fibers were analyzed. The experiment was carried out at three different levels, where the experimental temperature, nitric acid concentration, and ratio of sample weight to nitric acid solution was varied. The best combination of design parameters was determined to be a temperature of $90^{\circ} \mathrm{C}$, a concentration of $8 \mathrm{M}$, and a ratio of $4 \mathrm{~g}: 100 \mathrm{~mL}$. The recovered fibers experienced a 1.1\% loss in terms of single-fiber strength when processed at $90^{\circ} \mathrm{C}$, with a concentration of $8 \mathrm{M}$, and a ratio of $6 \mathrm{~g}: 100 \mathrm{~mL}$ [1].

Nakagawa et al. [12] applied the solvolysis process to recover carbon fibers from a variety of products manufactured with carbon fiber reinforced plastics (CFRP). In order to depolymerize the material, a potassium phosphate tribasic $\left(\mathrm{K}_{3} \mathrm{PO}_{4}\right)$ and benzyl alcohol (BYA) were used. The recovered fibers were used for the manufacture of recycled CFRP, and the properties thereof where compared to those of mass-produced glass fiber reinforced plastics (GFRP). The tensile elongation and strength of the recycled CFRP was found to be higher than that of the mass-produced GFRP, while the tensile modulus was equal [12]. Xu et al. [13] analyzed the characteristics of carbon fibers recovered via the solvolysis process to recover from carbon fiber/epoxy composite materials. The method applied consisted of a two-step solvolysis process. First, the material was treated with acetic acid, washed with acetone, and dried, with the purpose of obtaining a larger surface area. Secondly, the material was placed in a hermetic reactor with hydrogen peroxide (H2O2) and N,N-dimethylformamide (DMF). After the chemical treatment, the fibers were washed with acetone and dried. The experiment was repeated at temperatures ranging from $80^{\circ} \mathrm{C}$ to $100^{\circ} \mathrm{C}$, with different concentrations of $\mathrm{H} 2 \mathrm{O} 2$ and DMF, and for time periods ranging from 5 minutes to 120 minutes. The recovered fibers were subjected to tensile tests and analyzed via thermal gravimetric analysis (TGA), SEM, Raman spectroscopy, and X-ray diffraction (XRD). The tests revealed that the majority of the epoxy resin decomposed throughout the process, thus revealing smooth carbon fibers. In addition, the tensile strength of the recovered fibers was determined to be at least $95 \%$ of the tensile strength of virgin carbon fibers [13].

\section{Hydrolysis}

In the hydrolysis process, water is used as the solvent for the chemical degradation of the polymers $[6,8,9]$. Kao et al. [14] studied the effect of different experimental parameters of the hydrolysis process on the mechanical strength of glass fibers. For the study, $40 \times 40 \mathrm{~mm}$ sized samples of a fiberglass-based composites were immersed in sub-critical distilled water. The experimental temperature, duration, catalyst concentration, and water volume were varied in order to study their effect on the mechanical characteristics of the fibers. Prior to testing, the recovered fibers were washed with distilled water and acetone, and subsequently dried at air temperature. The mechanical strength of the fibers was found to have decreased postprocessing, as the recovered fibers only retain approximately $60 \%$ of the mechanical strength of virgin fibers. The water volume was found to have an effect on the cleanliness of the fibers-as reduced water volume resulted in a greater amount of resin remaining on the fibers. At a constant water volume, fibers processed at lower experimental temperatures for a longer period of time presented 
better mechanical properties [14]. Liu et al. [15] analyzed the effect of supplementing the solvolysis process with additives on the decomposition efficiency of CFRP. The two additives analyzed were phenol and potassium hydroxide (KOH). CFRP sheets were cut into samples of $1.5 \times 4 \mathrm{~cm}$ in size, placed in an autoclave, and submerged in different concentration combinations of subcritical water, phenol, and $\mathrm{KOH}$. The decomposition efficiency of the epoxy as well as the recovered fibers were studied. The maximum epoxy decomposition was achieved at a $1 / 10 / 100 \mathrm{~g} / \mathrm{g} / \mathrm{mL}$ ratio of $\mathrm{KOH}$ mass/phenol mass/water volume. The recovered fibers were characterized via single fiber tensile tests, SEM, and XPS. The surface composition of the recovered fibers was found to have slightly changed and the tensile strength was retained, when compared to virgin fibers [15].

The effect of several solvolysis process parameters on the recycling of carbon fiber reinforced epoxy composites were characterized by Oliveux et al. [16]. Using a design of experiments approach, the effect of process time, solvent volume, $\mathrm{H}_{2} \mathrm{O}_{2}$ concentration, and temperature on the yield of eliminated resin was studied. In addition, the mechanical properties of the recovered fibers were evaluated. The process achieved a resin removal rate of $79.3 \%$. The rate was increased to $95.3 \%$ with the use of $\mathrm{KOH}$ as a catalyst. The tensile strength of the recovered fibers was found to be between $90 \%$ and $98 \%$ when compared to the strength of virgin fibers [16]. Yuyan et al. [17] evaluated the effect of the solvolysis process temperature, time, pressure, feedstock, and catalyst on the potential for recovery of carbon fibers. Samples of $65 \mathrm{~mm} \times 6 \mathrm{~mm} \times 2 \mathrm{~mm}$ in size were placed in a batched autoclave and processed at various combinations of process parameters. Post-processing, the recovered fibers were washed with water and acetone. The recovered fibers were characterized via tensile strength tests, SEM, and atomic force microscopy (AFM). The recovered fibers were found to be clean and free of cracks and defects, while the tensile strength decreased by $1.8 \%$ when compared to virgin fibers. The factors with the greatest impact on the decomposition rate of the matrix were determined to be process temperature and pressure. The composition rate can be further increased by using a sulfuric acid solution as a catalyst [17].

The surface characteristics and mechanical strength of carbon fibers recovered via the pyrolysis process were further studied by Bai et al. [18]. Samples of carbon fiber epoxy reinforced composite materials were placed in oxygen in supercritical water and processed at $30 \pm 1 \mathrm{MPA}$ and $440 \pm 10^{\circ} \mathrm{C}$ for 25 to 35 minutes. Tensile tests, SEM, AFM, and XPS were used to characterize the recovered fibers. The fibers were found to be ideally recovered and retain original tensile strength at decomposition rates between 94 and 97wt. \% [18].

\section{Mechanical recycling}

In the mechanical recycling process the material to be recycled is reduced in size through multiple steps by shredding, crushing, or milling. The size reduction of the constituents increases the separation ability of the fibers and the resin. The first step consists of slow cutting or milling the material to approximately $100 \mathrm{~mm}$ of size. In order to reduce the volume of the material and thus facilitate its transport, metal inserts are extracted and separated from the composite material at the beginning stages of the process via magnetic fields. Once the metal inserts are extracted, the material is further downsized through the use high-speed mills. At the end of the downsizing process, the resulting material is sorted based on size through a combination of hydrocyclones, sieves, and shaking screens. Resulting coarser product is composed of a higher fiber content than the original material, while finer and powder-like product is composed of a higher filler and polymer content than the original material $[5,6]$. When ground to a very fine powder, the resulting material can be reused as a filler or reinforcement for the manufacturing of new composite materials $[8,19]$. Even though the mechanical recycling process has been explored for carbon fiber composites as well as fiberglass composites, the majority of the research has been centered around the mechanical recycling of fiberglass composites $[5,6,10]$.

Kouparitsas et al. [19] analyzed a mechanical grinding-sifting process to recover the fibers of epoxy-based carbon, glass, and aramid fiber composite materials, with the purpose of assessing the usability of the recovered fibers as reinforcing agents. The material to be recycled was cut into smaller pieces and fed into a mini granulator. The ground material was classified into two categories: fiber-rich fractions, later reused, and fractions of fines, composed of resin powder and microfibers. The recovered fibers were combined with virgin resin matrices to manufacture new thermoset composite samples. During tensile testing the samples manufactured with recovered fibers performed favorably when compared to samples manufactured with pristine virgin fibers [19]. Bernasconi et al. [20] researched the effect of using mechanically recycled fiberglass on the tensile strength of a glass fiber reinforced polyamide 6,6 with $35 \%$ by weight of fiberglass. Samples were manufactured using injection molding with different percentages of reprocessed fiberglass fibers, which were obtained via specimen grinding. The fiber length distribution and tensile strength of the samples was analyzed. During tensile testing it was found that as the reprocessed fiber content increased, the tensile strength as well as the elastic modulus decreased, and the strain at break increased [20]. Ogi et al. [21] studied the mechanical properties of an acrylonitrile-butadienestyrene (ABS) resin composite material reinforced with recycled carbon fiber reinforced plastic (CFRP) pieces. The recovered CFRP material was obtained via of crushing of a carbon/epoxy composite. Part of the recovered CFRP was further downsized via milling. The crushing process resulted in a blend of individual separated fibers and bundles of bigger sized fibers. Specimens with different weight concentrations of recovered CFRP and ABS were manufactured and mechanically tested.

The strength of the samples increased as the recovered crushed CFRP content increased, with the maximum strength obtained in 
samples with 50:50 weight concentration of recovered CFRP and ABS. The strength and modulus were found to be lower when using milled CFRP fibers instead of crushed CFRP fibers [21]. Takahashi et al. [22] evaluated the bending, tensile, and impact characteristics of continuous fiber reinforced thermoplastic (CFRTP) pellets manufactured with various amounts of recycled CFRP fibers per volume. The recycled material consisted of squares $1 \mathrm{~cm}$ in size obtained by mechanical crushing of CFRP. The pellets were used to manufacture JIS standard specimens and panels through injection molding for mechanical testing. While there was no significant different in flexural strength between specimens containing virgin and recycled fibers, the flexural strength varied depending on the recycled fiber concentration. The greatest flexural strength was obtained by specimens with a fiber concentration of $24 \%$ or less. Specimens manufactured with virgin materials obtained better results in tensile tests than samples containing recycled fibers. The characteristics of the manufactured panels was found to be dependent on fiber orientation. On panels with longitudinal fibers, the flexural modulus increased with fiber concentration. On panels with transversal fibers, the flexural modulus remained nearly constant as the fiber concentration changed. The failure strained was greater in samples with longitudinal fibers than in samples with transversal fiber alignment. However, in both panels the strain increased as the fiber concentration decreased. Based on these results, the recommended fiber concentration for the panels is $24 \%$. To assess the impact of multiple recycling, some specimens were subjected to four repetitions of crushing and injection molding. However, the characteristics of these samples did not vary significantly [22].

\section{Thermal recycling}

In thermal recycling heat is used to break down the different combustible components in composite materials, such as the polymer. The remaining non-combustible components can then be recovered and reused [23]. Additionally, as polymeric materials have high calorific values, heat and energy can be recovered in the recycling process from the combusted polymer [6,23]. Thermal recycling can be accomplished via multiple processes, namely combustion, fluidized bed, pyrolysis, and microwave heating $[5,8,10,24,25]$.

\section{Combustion}

In the combustion process, the scrap material is combusted with the purpose of energy recovery. The heat that is released when combusting organic materials, such as the ones as present in composite materials, can be recovered and converted into other types of energy $[5,6,8]$. However, several factors limit the amount of energy that can be recovered when combusting composite materials. First, the only organic component commonly present in composite materials such as sheet molding compounds (SMC) is the resin, comprising only approximately $35 \%$ of the weight of the materials. Furthermore, some fillers absorb energy during the combustion process, as they need high temperatures to decompose. In addition, some SMC contain fire retardants. Lastly, the combustion process results in solid residue, which is not suitable for reuse [8].

\section{Fluidized bed}

The fluidized bed process, or fluidized bed combustion (FBC), utilizes a bed with silica sand and heated air to separate the filler and fibers from the resin particles. The air is heated to $450-500^{\circ} \mathrm{C}$, allowing to transport and collect for further use the sand from the bed together with the non-organic materials - the fibers and the fillers-while volatilizing the organic materials in compositessuch as the resin $[8,23,26]$. The clean fibers are recovered and separated from the gas stream via a cyclone. After separation of the fibers, the gas stream is reheated to $1,000^{\circ} \mathrm{C}$ in a secondary combustion chamber to fully combust leftover organic polymer material $[23,26]$. Reheating the gas stream also provides an opportunity to recover energy from the polymers in the form of heat. The fluidized bed process is applied in several industries, as it allows for good heat transfer between solids and gases, good mixing, and operates at temperatures that can be properly controlled [27]. Initially research of the fluidized bed process focused on the recovery of glass fiber composites, however, the area has expanded to include the recovery of carbon fibers. As carbon fiber has a higher value compared to fiberglass, when a small volume of fibers is to be recovered, the recycling of carbon fiber can be more economical. The recovered fibers have the potential to be used for the fabrication of molding compounds, as well as tissue and veil type products $[23,26]$. The main limiting factors in reusing recovered fibres from the fluidized bed process to are the discontinuity and flocculating nature of the recovered fibres [26].

Yip et al. [23] studied the quality characteristics, in terms of tensile properties and fiber surface chemistry, of carbon fibers recovered via the fluidized bed process. The bed temperature and fluidizing velocity were identified to be the main factors to impact the efficiency and performance of the process. The fibers were recovered in the form of short and randomly oriented filaments. Some degradation was observed on the recovered fibers when compared to virgin fibers, however, they were found to have similar properties. Consequently, Yip et al. [23] concluded that the recovered fibers have the potential to be partially or fully reused as a replacement of short virgin carbon fibers [23]. Pickering et al. [25] applied the fluidized bed process to recover fibers from a sheet molding compound (SMC) fabricated with chopped E-glass fibers, an E-glass filament wound pipe, and an E-glass/polyester automobile body panel. Before the sample materials were fed into the fluidized bed, they were subjected to a size reduction process. The main factors affecting the process were identified to be the bed temperature, fluidizing velocity, secondary air flow and composite feed rate. The fluidizing bed temperature and fluidizing velocity were maintained constant for all materials, while the remaining operating parameters were varied depending on the material being processed. The process was repeated with bed temperatures of $450^{\circ} \mathrm{C}, 550^{\circ} \mathrm{C}$, and $650^{\circ} \mathrm{C}$, to analyze the effect 
of increasing temperature on fiber strength. Fiber strength was found to decrease as the fluidized bed operating temperature increased, supporting results from previous studies.

In addition, Pickering et al. [25] fabricated veils using different concentrations of recovered and virgin fibers, with the purpose of studying their strength characteristics. The veil strength was found to decrease as the recovered fiber concentration increased. Furthermore, fiber length and fiber content in addition to fiber strength, were identified to have an impact on veil strength [26]. Kennerley et al. [27] designed a fluidized bed test rig to recover glass fibers from a polyester based SMC. The bed was operated at different fluidizing velocities, ranging from $1.3 \mathrm{~m} / \mathrm{s}$ to $1.7 \mathrm{~m} / \mathrm{s}$, and at different temperatures, ranging from $450^{\circ} \mathrm{C}$ to $650^{\circ} \mathrm{C}$. The recovered glass fibers were contaminated with up to $60 \%$ with fillers. Most of contamination is mixed in with the fibers and can be washed away, only a small amount of the contamination is attached to the fibers. The fluidizing velocity did not impact the strength of the recovered fibers at the temperatures recorded. On the other hand, fluidizing bed temperature was observed to have an inverse relationship to fiber strength-as the fluidizing bed temperature increased, fiber strength decreased. Furthermore, polyester dough molding compound (DMC) was researched as a possible application for the recovered fibers. For the manufacture of DMC samples, a mixture of various levels of virgin fiber and recovered fibers from the fluidized bed process at $450^{\circ} \mathrm{C}$ were used. Before being used for the manufacture of DMC samples, the recovered samples were filtered, heat treated, washed, and rinsed to remove loose remaining filler material, organic materials, and filler particles respectively.

Additionally, to improve fiber to matrix boding, the fibers were treated with a polyester-compatible solution. During testing of the DMC samples, it was observed that an increase in the amount of recovered fibers resulted in a decrease in the flexural and tensile strength, strain at peak load, and impact strength. The flexural and tensile stiffness remained unaffected by the proportion of recovered fibers. The unaffected flexural and tensile stiffness of the samples is attributed to the unchanged fiber glass stiffness after the fluidized bed process, while the decrease in flexural and tensile strength is attributed to the weakened fibers after the fluidized bed process. Nevertheless, the properties of the DMC were determined to remain unaffected with up to a $50 \%$ concentration of recovered glass fibers [27].

\section{Pyrolysis}

In the pyrolysis thermal recycling method, the organic molecules in composite materials are decomposed in an inert atmosphere [28]. In the heated reduced oxygen environment, the resin together with the fillers drop away from the fibers, yielding a fiber-only output [25]. The pyrolysis process is being used to recycle composite materials by various companies worldwide. Examples include CFK Valley Stade Recycling GmbH \& Co. KG and HADEG Recycling in Ltd. in Germany, ELG Carbon Fibre Ltd. in the UK, Karborek S.p.a. in Italy, Japan Carbon Fiber Manufacturers
Association (JCMA) in Japan, and Carbon Conversions in the United States [28]. The pyrolysis process presents multiple advantages. First, the recovered fibers retain between $80 \%$ and $90 \%$ of the mechanical properties form the virgin materials. In addition, no chemicals are required since solely heat is used to separate the matrix from the fibers [25]. However, the pyrolysis recycling process also presents numerous drawbacks-there is a potential of char deposition on recovered fibers, the properties of the recovered material are highly dependent on the process parameters, and the process exhaust gases can be classified as environmentally hazardous [28]. Most of the research of the pyrolysis process focuses on the recovery of carbon fibers [10]. Meyer et al. [29] researched the effect of different parameters of the pyrolysis process on CFRP-based aircraft manufacturing prepreg waste materials, with the objective of optimizing the process. The pyrolysis process was evaluated at different operating temperatures and in two different environments, namely in nitrogen and synthetic.

The recovered fibers were analyzed through single fiber tensile tests, Raman spectroscopy and SEM. In addition, their electrical properties were measured. The results showed that the recovered fibers were similar to virgin fibers in terms of their properties. Furthermore, new composite materials were manufactured with the recovered fibers for mechanical and electrical testing. The recovered fibers were found to meet the demands of the automotive industry. Additionally, the potential of the fibers recovered through the pyrolysis process can be increased by additional surface treatments [29]. Cunliffe et al. [30] explored the effect of the temperature of the pyrolysis process on the product mass balance, gas composition, the nature of the condensable products, and the strength of the recovered fibers. Seven samples of with different compositions of composite plastic wastes were analyzed. The pyrolysis process was operated with nitrogen gas at temperatures ranging from $350^{\circ} \mathrm{C}$ to $800^{\circ} \mathrm{C}$. The samples were held at their respective temperature for 60 minutes. As a result, oils, waxes, and solids are obtained. The solids are formed by a mixture of fibers, fillers, and char. Two methods are tested to recover the fiber from the pyrolysis solids. First, a mechanical process, where the light pressure is applied to expose the fibers, which are then pulled away from the char. When the mechanical process is employed, a light char coating remains on the fibers. The second process consists of burning off the remaining char.

When burning heating the remaining solids at $450^{\circ} \mathrm{C}$, all char is oxidized, resulting in clean fibers. The fiber recovery affected the strength of the recovered fibers. Nevertheless, the recovered glass fibers retained between $50 \%$ and $60 \%$ of the virgin glass fiber tensile strength when subjected to pyrolysis temperatures of $400^{\circ} \mathrm{C}$ to $650^{\circ} \mathrm{C}$. At higher temperatures, the tensile strength decreased drastically [30]. Fibers recovered via the pyrolysis process were used by Szpieg et al. [31] to manufacture a composite material based on recycled polypropylene (rPP) and recycled carbon fibers $(\mathrm{rCF})$. The recovered carbon fibers were obtained from HADEG Recycling GmbH. The original material consisted of 
aircraft industry carbon fiber waste. The new composite material was manufactured using papermaking techniques, where the recovered fibers were dispersed in water, formed into mats, and pressed into a sandwich with layers of rPP. The new material was characterized in terms of fiber length, void content, plasticity, viscoelasticity, and tensile strength [31].

\section{Microwave}

In the microwave thermal recycling method, the material to be recycled is heated through the use of microwaves. The objective of the process is to recover both, clean fibers and the chemical polymers. The process has the potential of requiring less energy than other thermal processes [24]. Lester et al. [24] developed a microwave recycling method to recover clean fibers from a carbon fiber and epoxy resin composite material. The microwave was filled with nitrogen to produce an inert atmosphere and prevent the fibers from combusting during the heating process. The recovered fibers were characterized as relatively clean and long, with small remains of polymer and slight topological changes. The modulus of the recovered fibers was reduced compared to virgin fibers and fibers recovered via the fluidized bed process. However, the recovered fibers presented a higher strength retention than fibers recovered through the fluidized bed process [24].

\section{Conclusion}

Composite recycling methods in their current state are not mature enough to respond to the high demand of efficient and effective composite recycling. Worldwide, only a limited number of companies offer composite recycling services. Even though the recycling methodologies explored in this review were mostly able to recover fibers with properties similar to those of virgin fibers, a large amount of the studies described were performed on a smaller, laboratory scale. In addition to examining the properties of the recovered fibers, it is important to also consider the cost and energy requirements of the recycling methods examined and of large-scale recycling operations. Few studies in this area have been performed to date by McConell [2], Pickering et al. [26], Howarth et al. [32], Jody et al. [33], and Suzuki \& Takahashi [34]. Similarly, the recycling effort does not end after the fibers are recovered. Additional research into possible applications of the recovered materials, both in their original fields and for alternative uses, such as performed by Pickering et al. [26], Kennerley et al. [27], and Perry et al. [35] is required.

\section{References}

1. Liu Y, Meng L, Huang Y, Du J (2004) Recycling of carbon/epoxy composites. J Appl Polym Sci 94(5): 1912-1916.

2. McConell VP (2010) Launching the carbon fibre recycling industry Reinforced Plastics 54(2): 33-37.

3. Giulvezan G, Carberry B (2003) Composite recycling and disposal: an environmental R\&D issue. Boeing Environmental Technotes 8(4): 1-4.

4. Koblen I, Talpas L, Jurcak J (2012) Selected aspects of aviation equipment disposal issue. Review of the Air Force Academy (2): 102108.
5. Pickering SJ (2006) Recycling technologies for thermoset composite materials-current status. Compos Part A-Appl S 37(8): 1206-1215.

6. Asmatulu E, Twomey J, Overcash M (2014) Recycling of fiber-reinforced composites and direct structural composite recycling concept. J Compos Mater 48(5): 593-608.

7. Asmatulu E, Overcash M, Twomey J (2013) Recycling of aircraft: state of the art in 2011. J Ind Engineering, pp. 1-8.

8. Palmer JAT (2009) Mechanical recycling of automotive composites for use as reinforcement in thermoset composites-PhD Thesis. University of Exeter, pp. 1-224.

9. Yang Y, Boom R, Irion B, van Heerden DJ, Kuiper P, etal. (2012) Recycling of composite materials. Chem Eng Process 51: 53-68.

10. Rybicka J, Tiwari A, Leeke GA (2016) Technology readiness level assessment of composites recycling technologies. J Clean Prod 112: 1001-1012.

11. Jiang G, Pickering SJ, Lester EH, Turner TA, Wong KH, et al. (2009) Characterisation of carbon fibres recycled from carbon fibre/epoxy resin composites using supercritical n-propanol. Compos Sci Technol 69(2): 192-198.

12. Nakagawa M, Kuriya H, Shibata K (2009) Characterization of CFRP using recovered carbon fibers from waste CFRP. In Second International Symposium on Fiber Recycling, pp. 241-244.

13. Xu P, Li J, Ding J (2013) Chemical recycling of carbon fibre/epoxy composites in a mixed solution of peroxide hydrogen and $\mathrm{N}, \mathrm{N}$ dimethylformamide. Compos Sci Technol 82: 54-59.

14. Kao CC, Ghita OR, Hallam KR, Heard PJ, Evans KE (2012) Mechanical studies of single glass fibres recycled from hydrolysis process using sub-critical water. Compos Part A-Appl S 43(3): 398-406.

15. Liliuli12) Chemical recycling of carbon fibre reinforced epoxy resin composites in subcritical water: synergistic effect of phenol and $\mathrm{KOH}$ on the decomposition efficiency. Polymer Degrad Stabil 97(3): 214220.

16. Oliveux G, Bailleul JL, Le Gal La Salle E, Lefevre N, Biotteau G (2013) Recycling of glass fibre reinforced composites using subcritical hydrolysis: reaction mechanisms and kinetics, influence of the chemical structure of the resin. Polymer Degrad Stabil 98(3): 785-800.

17. Yuyan L, Guohua S, Linghui M (2009) Recycling of carbon fibre reinforced composites using water in subcritical conditions. Mater Sci Eng A 520(1-2): 179-183.

18. Bai Y, Wang Z, Feng L (2010) Chemical recycling of carbon fibers reinforced epoxy resin composites in oxygen in supercritical water. Mater Design 31(2): 999-1002.

19. Kouparitsas CE, Kartalis CN, Varelidis PC, Tsenoglou CJ, Papaspyrides CD (2002) Recycling of the fibrous fraction of reinforced thermoset composites. Polym Composite 23(4): 682-689.

20. Bernasconi A, Rossin D, Armanni C (2007) Analysis of the effect of mechanical recycling upon tensile strength of a short glass fibre reinforced polyamide 6,6. Eng Fract Mech 74(4): 627-641.

21. Ogi K, Nishikawa T, Okano Y, Taketa I (2007) Mechanical properties of ABS resin reinforced with recycled CFRP. Adv Compos Mater 16(2): 181-194.

22. Takahashi J, Matsutsuka N, Okazumi T, Uzawa K, Ohsawa I, et al. (2007) Mechanical properties of recycled CFRP by injection molding method. In 16th International Conference of Composite Materials, Japan, pp. 1-6.

23. Yip HLH, Pickering SJ, Rudd CD (2002) Characterisation of carbon fibres recycled from scrap composites using fluidised bed process. Plast Rubber Compos 31(6): 278-282. 
24. Lester E, Kingman S, Wong KH, Rudd C, Pickering S, et al. (2004) Microwave heating as a means for carbon fibre recovery from polymer composites: a technical feasibility study. Mater Res Bull 39(10): 15491556.

25. Marsh G (2008) Reclaiming value from post-use carbon composite. Reinforced Plastics 52(7): 36-39.

26. Pickering SJ, Kelly RM, Kennerley JR, Rudd CD, Fenwick NJ (2000) A fluidised-bed process for the recovery of glass fibres from scrap thermoset composites. Compos Sci Technol 60(4): 509-523.

27. Kennerley JR, Kelly RM, Fenwick NJ, Pickering SJ, Rudd CD (1998) The characterisation and reuse of glass fibres recycled from scrap composites by the action of a fluidised bed process. Compos Part A-Appl S 29(7): 839-845.

28. Pimenta S, Pinho ST (2011) Recycling carbon fibre reinforced polymers for structural applications: technology review and market outlook Waste Manage 31(2): 378-392.

29. Meyer LO, Schulte K, Grove-Nielsen E (2007) Optimisation of a pyrolysis process for recycling CFRP's. In 16th International Conference of Composite Materials, Japan, pp. 1-2.

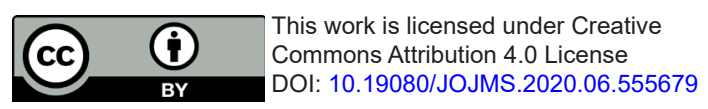

30. Cunliffe AM, Jones N, Williams PT (2003) Pyrolysis of composite plastic waste. Environ Technol 24(5): 653-663.

31. Szpieg M, Wysocki M, Asp LE (2011) Mechanical performance and modelling of fully recycled modified CF/PP composite. J Compos Mater 46(12): 1503-1517.

32. Howarth J, Mareddy SS, Mativenga PT (2014) Energy intensity and environmental analysis of mechanical recycling of carbon fibre composite. J Clean Prod 81: 46-50.

33. Jody BJ, Daniels EJ, Pomykala JA (1999) Thermal decomposition of PMC for fiber recovery. Argonne National Lab, USA, pp. 1-6.

34. Suzuki T, Takahashi J (2005) Prediction of energy intensity of carbon fiber reinforced plastics for mass-produced passenger cars. In Ninth Japan International SAMPE Symposium, pp. 1-6.

35. Perry N, Mantaux O, Leray D, Lorriot T (2010) Composite recycling: design for environment approach requirements. In Proceedings of IDMME -Virtual Concept 2010, France, pp. 1-4.

Your next submission with Juniper Publishers
will reach you the below assets
- Quality Editorial service
- Swift Peer Review
- Reprints availability
- E-prints Service
- Manuscript Podcast for convenient understanding
- Global attainment for your research
- Manuscript accessibility in different formats
( Pdf, E-pub, Full Text, Audio)
- Unceasing customer service
Track the below URL for one-step submission
https://juniperpublishers.com/online-submission.php

